\title{
Perfil das infecções de sítio cirúrgico em ginecologia e obstetrícia em um hospital público de ensino
}

\author{
Profile of surgical site infections in gynecology and obstetrics in a public teaching hospital \\ Perfil de las infecciones del sitio quirúrgico en ginecología y obstetricia en un hospital universitario \\ público
}

Recebido: 25/10/2021 | Revisado: 01/11/2021 | Aceito: 05/11/2021 | Publicado: 09/11/2021

\author{
Manoela Cristina Berticelli \\ ORCID: https://orcid.org/0000-0003-3570-1336 \\ Universidade Estadual do Oeste do Paraná, Brasil \\ E-mail: manu.berticelli@gmail.com \\ Fabiana Gonçalves de Oliveira Azevedo Matos \\ ORCID: https://orcid.org/0000-0002-5283-5363 \\ Universidade Estadual do Oeste do Paraná, Brasil \\ E-mail: fabianamatos@ hotmail.com \\ Débora Cristina Ignácio Alves \\ ORCID: https://orcid.org/0000-0001-6892-366X \\ Universidade Estadual do Oeste do Paraná, Brasil \\ E-mail: dcialves@gmail.com \\ Graziela Braun \\ ORCID: https://orcid.org/ 0000-0003-4372-8256 \\ Universidade Estadual do Oeste do Paraná, Brasil \\ E-mail: grazibraun@yahoo.com.br \\ Maria Júlia Navarro Kassim \\ ORCID: https://orcid.org/0000-0001-9753-2131 \\ Universidade Estadual do Oeste do Paraná, Brasil \\ E-mail: mjkassim_enfermagem@hotmail.com
}

\begin{abstract}
Resumo
O objetivo do estudo foi identificar as infecções de sítio cirúrgico relacionadas a procedimentos ginecológicos e obstétricos. Método: estudo documental, transversal, retrospectivo e quantitativo. As ISC foram analisadas entre março de 2017 e março de 2019. As variáveis de estudo foram: idade, planos anatômicos acometidos, procedimento cirúrgico, critério de confirmação, microrganismo isolado, desfecho do caso e tempo de retorno ambulatorial. A análise descritiva foi utilizada para exploração dos dados. Resultados: foram notificados 322 casos de ISC, sendo $41 \%$ ginecológico-obstétricos. A taxa de ISC foi calculada em 1,5\%. A faixa etária mais acometida foi de 21 a 30 anos, com predominância de infecções incisionais superficiais (63\%), diagnosticadas clinicamente (97\%), com destaque para parto cesáreo (90\%). A maioria das pacientes (68\%) retornou para avaliação pós-operatória em até dez dias, com alta clínica (100\%). Conclusão: apesar da divulgação dos critérios diagnósticos, as ISC se mostraram elevadas no hospital em estudo, indicando a necessidade de adotar medidas protocolares preventivas.
\end{abstract}

Palavras-chave: Infecções; Infecção da ferida cirúrgica; Infecção puerperal; Monitoramento epidemiológico; Hospitais universitários.

\begin{abstract}
The aim of the study was to identify surgical site infections related to gynecological and obstetric procedures. Method: documentary, cross-sectional, retrospective and quantitative study. The SSI were analyzed between March 2017 and March 2019. The study variables were: age, anatomical planes involved, surgical procedure, confirmation criteria, isolated microorganism, case outcome and time of outpatient return. Descriptive analysis was used for data exploration. Results: 322 cases of SSI were reported, $41 \%$ of which were gynecological-obstetric. The ISC rate was calculated at $1.5 \%$. The most affected age group was 21 to 30 years, with a predominance of superficial incisional infections (63\%), clinically diagnosed (97\%), especially cesarean delivery (90\%). Most patients (68\%) returned for postoperative evaluation within ten days, with clinical discharge (100\%). Conclusion: despite the disclosure of diagnostic criteria, the SSI were high in the hospital under study, indicating the need to adopt preventive protocol measures.
\end{abstract}

Keywords: Infections; Surgical wound infection; Puerperal infection; Epidemiological monitoring; University hospitals. 


\section{Resumen}

El objetivo del estudio fue identificar infecciones del sitio quirúrgico relacionadas con procedimientos ginecológicos y obstétricos. Método: estudio documental, transversal, retrospectivo y cuantitativo. Las ISQ se analizaron entre marzo de 2017 y marzo de 2019. Las variables de estudio fueron: edad, planos anatómicos involucrados, procedimiento quirúrgico, criterios de confirmación, microorganismo aislado, desenlace del caso y tiempo de retorno ambulatorio. Se utilizó análisis descriptivo para la exploración de datos. Resultados: se notificaron 322 casos de ISQ, el $41 \%$ de los cuales fueron ginecológico-obstétricos. La tasa de ISC se calculó al 1,5\%. El grupo de edad más afectado fue el de 21 a 30 años, con predominio de infecciones incisionales superficiales (63\%), diagnosticadas clínicamente $(97 \%)$, especialmente parto por cesárea $(90 \%)$. La mayoría de los pacientes $(68 \%)$ regresaron para evaluación postoperatoria dentro de los diez días, con alta clínica (100\%). Conclusión: a pesar de la divulgación de los criterios diagnósticos, las ISQ fueron elevadas en el hospital en estudio, lo que indica la necesidad de adoptar medidas de protocolo preventivo.

Palabras clave: Infecciones; Infección de heridas quirúrgicas; Infección puerperal; Monitoreo epidemiológico; Hospitales universitarios.

\section{Introdução}

As Infecções Relacionadas à Assistência em Saúde (IRAS) são definidas como quaisquer infecções adquiridas após a admissão do paciente ou após a alta, quando relacionadas com a internação ou procedimentos hospitalares (Scardoni et al., 2020). As IRAS são consideradas um problema de saúde pública tanto no Brasil quanto no mundo, e classificadas em quatro topografias principais: Infecção de Trato Urinário (ITU), Infecção de Sítio Cirúrgico (ISC), Infecção de Trato Respiratório (ITR) e Infecção Primária de Corrente Sanguínea (IPCS) (Anchieta et al., 2019).

Entre as IRAS, a ISC é o terceiro tipo de infecção mais frequente (Martins et al., 2012; Cruz et al., 2013). A ISC é definida como o processo inflamatório e infeccioso da ferida ou cavidade operada, com presença de drenagem de secreção purulenta, confirmada clínica ou laboratorialmente, podendo envolver somente a incisão cirúrgica ou as estruturas adjacentes manipuladas durante a cirurgia (Cruz et al, 2013). De acordo com o "Center for Disease Control and Prevention" (CDC), as ISC são classificadas conforme os planos anatômicos acometidos, sendo elas: Incisional Superficial (IS), Incisional Profunda (IP) e Órgãos e Cavidades (OC) (Martins et al., 2012; Cruz et al., 2013).

Em 2017, a Agência Nacional de Vigilância Sanitária (ANVISA) publicou os critérios diagnósticos de IRAS com base nas diretrizes do $C D C$, especificando alguns sinais e sintomas essenciais para o diagnóstico das ISC, tais como: drenagem purulenta da incisão cirúrgica; cultura positiva de secreção ou tecido/órgão/cavidade (obtido assepticamente); incisão superficial aberta pelo cirurgião na vigência de algum dos seguintes sinais ou sintomas: dor, edema local, aumento da sensibilidade, hiperemia ou calor; ou diagnóstico de infecção estabelecido pelo cirurgião ou pelo médico assistente (Brasil 2017a; Brasil 2019b).

Consideradas um evento adverso evitável, as ISC têm representação significativa entre as complicações decorrentes do ato cirúrgico e podem ocorrer em até $20 \%$ dos pacientes submetidos a qualquer intervenção cirúrgica (Souza et al., 2018), acarretando aumento da morbimortalidade dos pacientes cirúrgicos.

Os fatores de risco associados ao desenvolvimento das ISC podem estar relacionados ao próprio procedimento cirúrgico (potencial de contaminação da ferida operatória e porte cirúrgico) ou relacionados a fatores intrínsecos e extrínsecos dos próprios pacientes (idade, índice glicêmico, índice de massa corporal, fatores nutricionais, uso de antibióticos, medicamentos imunossupressores, tabagismo, doença crônica preexistente, realização do banho pré-operatório, tricotomia e antissepsia do campo cirúrgico) (Padoveze \& Fortaleza, 2014).

Pacientes que desenvolvem ISC têm maior custo com o tratamento e maior tempo de internação. Estudos recentes norte-americanos mostraram um acréscimo de 6,5 dias de internação, com um custo de mais de três mil dólares, cinco vezes mais chances de reinternação após alta hospitalar (Costa, 2019) e maior risco de morte, quando comparados àqueles que não desenvolveram infecção relacionada ao procedimento cirúrgico (Padoveze \& Fortaleza, 2014). O rastreamento e a 
monitorização dos pacientes submetidos a procedimentos cirúrgicos são de responsabilidade do Serviço de Controle de Infecção Hospitalar (SCIH), utilizando-se de critérios padronizados e análise sistemática dos dados coletados de forma a favorecer a tomada de decisão clínica. Um programa de vigilância em saúde efetivo pode diminuir significativamente as taxas de ISC, o qual é um importante indicador da atuação dos profissionais de saúde envolvidos na assistência (Souza et al., 2018).

Entre as cirurgias ginecológicas, a cesariana é uma das mais frequentes no Brasil (Cruz et al., 2013), sendo o país com a maior taxa de parto cesáreo do mundo (56,6\%), ultrapassando as recomendações da Organização Mundial da Saúde (OMS), que preconizam uma taxa máxima de 15\% de cesarianas (Brasil, 2016c).

A realização do parto cesáreo de forma não criteriosa apresenta riscos tanto para a gestante como para o recémnascido, com destaque para as infecções puerperais. Apesar do avanço das técnicas cirúrgicas e de limpeza e esterilização dos instrumentais cirúrgicos, cerca de $7 \%$ das mulheres submetidas à cesariana no Brasil evoluem com infecção pós-parto (Carvalho et al., 2010).

O fenômeno que desencadeou a realização do presente estudo foi o elevado número de infecções puerperais notificadas na referida instituição. Diante desse cenário, a pergunta que norteou o desenvolvimento da pesquisa foi: "Qual o perfil das ISC que acometem as pacientes da clínica ginecológica e obstétrica do hospital em estudo?”. Para responder tal questionamento, o objetivo do estudo foi identificar as ISC relacionadas a procedimentos ginecológicos e obstétricos notificadas em um hospital público de ensino.

\section{Metodologia}

Estudo documental, transversal, retrospectivo, com análise quantitativa dos dados. A pesquisa foi realizada em um hospital público de ensino, localizado na região oeste paranaense. A instituição pesquisada possui 212 leitos para atendimento exclusivo de pacientes do Sistema Único de Saúde (SUS). Por ser referência em gestação de alto risco, atende pacientes dos 25 municípios que compõem a 10ª Regional de Saúde do Paraná (Paraná, 2018). O centro obstétrico (CO) do referido hospital possui três salas cirúrgicas onde são realizados, em média, 10 partos/dia.

A população de estudo foi constituída por 131 casos de ISC relacionados à clínica ginecológica e obstétrica, correspondendo à totalidade (100\%) de casos notificados pelo SCIH no período de março de 2017 a março de 2019. O período de abrangência do estudo foi determinado a partir da adoção institucional dos critérios diagnósticos de IRAS definidos pela ANVISA (Brasil, 2017a).

A coleta de dados foi realizada nos meses de setembro e outubro de 2019. A extração dos dados foi realizada manualmente, a partir da planilha interna de controle de IRAS alimentada pelos membros do SCIH.

Foram analisadas as seguintes variáveis referentes às ISC: idade (em anos), planos anatômicos acometidos, procedimento cirúrgico, critério de confirmação da ISC, microrganismo isolado, desfecho e tempo (em dias) decorrido entre o procedimento cirúrgico e o retorno ambulatorial.

Os dados foram inseridos em planilhas do software Microsoft Office Excel, versão 16.12, tabulados e submetidos à análise estatística descritiva, sendo apresentados na forma de frequência absoluta e relativa.

A pesquisa atendeu todos os preceitos éticos da Resolução 466/12 e foi aprovada pelo Comitê de Ética e Pesquisa com Seres Humanos, parecer n ${ }^{\circ}$ 3.062.301, CAAE: 50066815.8.0000.0107.

\section{Resultados}

No período de setembro a março de 2019, foram realizados 19.451 (100\%) procedimentos cirúrgicos, dos quais 8.458 (43\%) relativos a procedimentos ginecológicos ou obstétricos. 
Nesse período, o SCIH da instituição pesquisada notificou 1.541 casos de IRAS, sendo que $21 \%$ (n=322) dos casos notificados eram relativos a ISC. Do total das ISC, $41 \%(\mathrm{n}=131)$ dos casos eram decorrentes de procedimentos ginecológicos ou obstétricos, correspondendo a uma taxa de ISC de 1,5\% na referida especialidade.

A Tabela 1 evidencia a distribuição das variáveis das ISC decorrentes de procedimentos ginecológicos ou obstétricos no hospital em estudo.

Tabela 1 - Distribuição das variáveis relacionadas às ISC decorrentes de procedimentos ginecológicos ou obstétricos em um hospital público de ensino da região oeste do Paraná (n=131). Cascavel, 2019.

\begin{tabular}{|c|c|c|}
\hline Variáveis de estudo & $\mathbf{N}^{\mathbf{0}}$ & $\%$ \\
\hline \multicolumn{3}{|l|}{ Idade } \\
\hline 15 a 20 & 26 & 20 \\
\hline 21 a 30 & 76 & 58 \\
\hline 31 a 39 & 19 & 14,5 \\
\hline 40 a 49 & 05 & 04 \\
\hline 50 a 59 & 04 & 03 \\
\hline Mais de 60 & 01 & 0,5 \\
\hline \multicolumn{3}{|l|}{ Plano anatômico acometido } \\
\hline Infecção incisional superficial & 83 & 63 \\
\hline Infecção profunda & 19 & 15 \\
\hline Infecção de órgão/cavidade & 12 & 09 \\
\hline Outros (mais que um plano acometido) & 17 & 13 \\
\hline \multicolumn{3}{|l|}{ Procedimento cirúrgico } \\
\hline Parto cesáreo & 118 & 90 \\
\hline Parto normal com episiotomia & 07 & 05 \\
\hline Histerectomia & 05 & 04 \\
\hline Curetagem & 01 & 01 \\
\hline \multicolumn{3}{|l|}{ Critérios de confirmação diagnóstica } \\
\hline Clínicos & 127 & 97 \\
\hline $\begin{array}{l}\text { Laboratorialmente confirmados } \\
\text { Tempo de retorno (dias) }\end{array}$ & 04 & 03 \\
\hline 1 a 10 & 89 & 68 \\
\hline 11 a 20 & 34 & 26 \\
\hline Acima de 21 & 08 & 06 \\
\hline \multicolumn{3}{|l|}{ Desfecho do caso } \\
\hline Alta & 131 & 100 \\
\hline TOTAL & 131 & 100 \\
\hline
\end{tabular}

Fonte: Dados da pesquisa (2021).

A faixa etária mais frequente das pacientes acometidas foi de 21 a 30 anos (58\%). Houve predominância de infecções classificadas como incisionais superficiais $(63 \%)$ e diagnosticadas clinicamente $(97 \%)$. Entre os procedimentos cirúrgicos que evoluíram com ISC, o parto cesáreo foi o mais frequente (90\%) e a maioria das pacientes (68\%) retornou para avaliação ambulatorial em até 10 dias, sendo que todas as pacientes (100\%) evoluíram com alta clínica.

Dos 131 casos de ISC identificados, apenas quatro foram confirmados por cultura microbiológica laboratorial; foi 
identificado um caso de ISC por Staphylococcus aureus (25\%); um caso por Pseudomonas aeruginosa (25\%); um caso por Candida albicans (25\%); e um caso por Escherichia coli (25\%).

\section{Discussão}

As ISC ocuparam o segundo lugar entre as IRAS notificadas $(\mathrm{n}=322 ; 21 \%)$ no hospital em estudo, reforçando a necessidade da avaliar os riscos potenciais relacionados às ISC (Prates, 2018), visto que nos países em desenvolvimento, podem acometer até um terço de todos os pacientes submetidos à procedimentos cirúrgicos, ocupando segundo lugar dentre as IRAS na Europa e Estados Unidos (Anderson et al, 2014) e terceiro lugar em nível nacional (Brasil, 2019b).

As ISC relacionadas a procedimentos obstétricos no Brasil são frequentes e colocam em risco a saúde materna. Existem poucos dados no âmbito nacional sobre o panorama das infecções puerperais, o que limita a comparação dos achados entre as diferentes localidades (Brasil, 2017d). Estima-se que entre 1 e 7,2\% das mulheres submetidas a procedimentos obstétricos são acometidas por alguma infecção puerperal, que consiste na terceira maior causa de óbito materno no Brasil (Lima et al., 2014).

Em 2014, foi estabelecido pelo Programa Nacional de Prevenção de Infecção Relacionada à Assistência à Saúde (PNPCIRAS) que a taxa de ISC cesárea (ISC-PC) fosse um dos indicadores nacionais do Programa (Brasil, 2017d). Em 2015, a incidência nacional de ISC-PC foi calculada em 1,1\%. No presente estudo, a taxa de ISC relacionada a procedimentos ginecológicos e obstétricos foi calculada em 1,5\%, similar ao encontrado em outros estados brasileiros como Rio de Janeiro, Bahia e Santa Catarina, porém acima da média paranaense $(0,7 \%)$, o que reforça a necessidade de revisão das medidas de controle de infecção adotadas na referida instituição (Brasil, 2015e). No entanto, um ponto que merece destaque é que a instituição investigada realiza vigilância pós-alta dos pacientes cirúrgicos por meio das consultas de retorno ambulatorial e de acordo com a literatura, os índices de ISC costumam ser maiores em hospitais que realizam vigilância pós-alta (Brasil, 2015e), devido ao menor número de subnotificações.

Com relação à idade das pacientes notificadas com ISC, a faixa etária de 21 a 30 anos foi a mais frequente (n=76; 58\%). A média de idade das pacientes foi de 27,42 anos, com variação de 15 a 68 anos. Tais resultados corroboram com estudo realizado no Distrito Federal (Carvalho et al., 2014), que identificou a prevalência de infecções ginecológicas em mulheres com idade entre 20 e 29 anos.

Assim como descrito em alguns estudos(Feitosa et al., 2014; Brasil, 2015e), a atual investigação identificou que as infecções incisionais superficiais $(n=83 ; 63 \%)$ (que acometem a pele e o tecido subcutâneo) foram as mais frequentes. Por parecerem mais simples, elas podem ser subnotificadas e subtratadas, podendo evoluir com maior gravidade.

Dentre os procedimentos cirúrgicos que apresentaram ISC, a cesárea foi a mais frequente ( $\mathrm{n}=118,90 \%)$. O aumento da morbidade e da mortalidade materna e neonatal está diretamente relacionado à maior incidência de partos cesáreos (Cunha, 2018), sendo que a sepse puerperal pode causar diversas complicações, desde doenças pélvicas inflamatórias, infertilidade e até óbito materno (Martins et al., 2010; Say et al., 2014). Alguns fatores de risco como trabalho de parto prolongado, excesso de toque vaginal, presença de mecônio, realização de cesárea de urgência, idade avançada da gestante e tempo cirúrgico prolongado também contribuem para o aumento da morbimortalidade materna.

No Brasil, as ISC relativas aos procedimentos puerperais possuem grande relevância epidemiológica, visto que o número de cesarianas realizadas no país ultrapassa a taxa recomendada pela OMS (15\%) (Cruz et al., 2013) e de acordo com o Ministério da Saúde, 41,9\% dos partos realizados pelo SUS no ano de 2017 foram cesarianas (Brasil, 2018f). O controle das infecções puerperais tem papel fundamental como um importante fator de diminuição da morbimortalidade perinatal e materna, uma vez que as pacientes que desenvolvem quadros infecciosos costumam apresentar períodos de recuperação mais longos, com maior risco de complicações e outros agravos associados (Cruz et al., 2013). As infecções aumentam os custos 
hospitalares com testes diagnósticos e tratamentos, prolongam o uso de antimicrobianos, aumentam o tempo de internação e as chances de reinternamento (Costa et al., 2019).

O processo infeccioso depende da espécie, virulência e resistência do agente patogênico aos agentes antimicrobianos (Brasil, 2018). O ambiente hospitalar é colonizado por inúmeros microrganismos, e grande parte deles é multirresistente à antibioticoterapia, fazendo com que a própria internação hospitalar seja fator de risco para o desenvolvimento de IRAS (Ferraz et al., 2016).

É possível observar que quase a totalidade das ISC relacionadas a procedimentos ginecológicos ou obstétricos foram diagnosticadas clinicamente $(\mathrm{n}=127 ; 97 \%)$, por meio dos critérios nacionais estabelecidos para diagnosticar as IRAS (Brasil 2017a; Brasil 2019b). Tais critérios devem ser adotados por todos os serviços de saúde, com a finalidade de padronizar as ações diagnósticas e possibilitar a comparação dos achados (Brasil 2017a; Brasil 2019b). Apenas 3\% ( $\mathrm{n}=4$ ) das ISC relacionadas aos procedimentos ginecológicos ou obstétricos foram confirmadas laboratorialmente, expondo a fragilidade da instituição, que é de não adotar como rotina a coleta de material microbiológico de feridas para análise laboratorial, uma vez que tal prática favorece a escolha da terapêutica adequada e previne a resistência antimicrobiana (Costa, 2019). A associação entre diagnóstico clínico e laboratorial favorece uma assistência assertiva e segura ao paciente (Mota, 2010).

Os resultados das culturas microbiológicas identificaram a presença de Staphylococcus aureus $(\mathrm{n}=1 ; 25 \%)$, Candida albicans $(\mathrm{n}=1 ; 25 \%)$, Escherichia coli $(\mathrm{n}=1 ; 25 \%)$ e Pseudomonas aeruginosa $(\mathrm{n}=1 ; 25 \%)$ nos exsudatos das feridas operatórias analisadas. Com exceção da Pseudomonas aeruginosa, os demais microrganismos são patógenos comumente encontrados nas culturas das feridas operatórias por pertencerem à própria microbiota humana (Santos et al., 2016).

Com relação ao tempo transcorrido entre o procedimento cirúrgico e a avalição pós-alta, $68 \%$ ( $\mathrm{n}=89)$ das pacientes com sinais e sintomas de ISC retornaram para avaliação ambulatorial em até 10 dias. O tempo médio transcorrido foi de 8,72 dias, com variação de um a 46 dias. A vigilância infecciosa vai desde a avaliação realizada em âmbito hospitalar até o pós-alta, por meio de acompanhamento ambulatorial, visita domiciliar ou contato telefônico realizado em até 30 dias após a alta hospitalar (Souza et al., 2018). É necessário estabelecer estratégias para identificação e acompanhamento dos pacientes submetidos a procedimentos cirúrgicos, considerando que a maioria das ISC é diagnosticada durante a vigilância pós-alta (Carvalho et al., 2017). No Brasil, a maioria dos hospitais limita a vigilância de ISC ao período de internação, não incluindo o acompanhamento por retorno ambulatorial, considerado um dos métodos mais eficientes para a avaliação do sítio cirúrgico, uma vez que a avaliação da ferida por profissional habilitado confere maior credibilidade do que a busca pelo telefone (Souza et al, 2018).

Quanto ao desfecho clínico, todas as pacientes que desenvolveram ISC relacionadas a procedimentos ginecológicos ou obstétricos $(\mathrm{n}=131,100 \%)$ apresentaram prognóstico favorável, evoluindo com alta clínica. Analisando tal indicador, é possível inferir que apesar da ocorrência do referido evento adverso (ISC), os casos notificados e tratados responderam bem à terapia prescrita, sem acarretar grandes danos fisiológicos às pacientes acometidas.

Apesar de o referido serviço realizar rigorosa busca ativa dos casos de IRAS, reconhecemos a possibilidade de haver subnotificação dos mesmos, se constituindo em uma limitação da pesquisa. Os resultados da pesquisa trazem importantes contribuições para a saúde pública, visto que descrevem o perfil das ISC relacionadas a procedimentos ginecológicos/obstétricos de uma determinada região e fornecem dados que permitem comparações com outras localidades. Da mesma forma, tais resultados permitem o planejamento de medidas gerenciais e assistenciais seguras no hospital investigado (controle do ambiente pré e pós-cirúrgico, controle de fatores extrínsecos do paciente cirúrgico, implantação de protocolos de cirurgia segura) como forma de prevenir as ISC. 


\section{Conclusão}

Os achados do estudo permitiram identificar o perfil das ISC relacionadas aos procedimentos ginecológicos e obstétricos notificadas em um hospital público de ensino. O escasso número de confirmações microbiológicas é um dado preocupante, pois a ausência de tal informação pode influenciar negativamente a terapêutica adotada.

A utilização dos critérios diagnósticos para as ISC de forma isolada não é suficiente para diminuir os índices de infecção na instituição investigada. Ressalta-se a necessidade de revisão dos protocolos assistenciais para o correto diagnóstico, manejo e prevenção de tais eventos. Ressalta-se também necessidade de revisar o cumprimento do checklist de cirurgia segura e fornecer informações adequadamente às pacientes sobre os cuidados com a ferida cirúrgica após a alta hospitalar.

Evidencia-se a necessidade de mais pesquisas sobre o assunto, com amostras significativas, visto que no processo de levantamento bibliográfico, identificou-se escassez no conteúdo bibliográficos.

\section{Referências}

Anchieta, D. W. Matos, F. G. O. A. Alves, D. C. I. Santos, R. P. Oliveira, J. L. C., \& Molin, T. D. (2019). Characterization of surgical site infections in a public teaching hospital in Cascavel, Paraná Vigil. Sanit. Debate, 7(3), 31-36.

Anderson, D. J. et al (2014). Strategies to prevent surgical site infections in acute care hospitals: 2014 UPDATE. Infect control hosp epidemiol., 35(6), 60527.

Brasil. (2015e) Agência Nacional de Vigilância Sanitária. Boletim Informativo: Segurança do Paciente e Qualidade em Serviços de Saúde nº 13. Avaliação dos indicadores nacionais de infecção relacionada à assistência à saúde e resistência microbiana ano de 2015. Brasília: ANVISA.

Brasil. (2016c) Portaria 306, de 28 de março de 2016. Aprova as Diretrizes de Atenção à Gestante: a operação cesariana. Ministério da Saúde.

Brasil. (2017a). Agência Nacional de Vigilância Sanitária. Critérios Diagnósticos de Infecções Relacionadas à Assistência à Saúde/Agência Nacional de Vigilância Sanitária.

Brasil. (2017d). Agência Nacional de Vigilância Sanitária. Medidas de Prevenção e Critérios Diagnósticos de Infecções Puerperais em Parto Vaginal e Cirurgia Cesariana, 2017d.

Brasil. (2018f). Ministério da Saúde fará monitoramento online de partos cesáreos no país. Agencia Saúde.

Brasil. (2019b). Agência Nacional de Vigilância Sanitária. Nota Técnica GVMS/GGTES 03/2019. Critérios Diagnósticos das Infecções Relacionadas à Assistência à Saúde. Brasília.

Carvalho, I. C. R. Medeiros, G. O., \& Souza, L. M. (2010). Proposal of establishment of a nursing protocol for the care of patients with abscess wall after cesarean section. Comum. Ciênc. Saúde; 21(1), 9-20.

Carvalho, R. L. R. Campos, C. C. Franco, L. M. C. Rocha, A. D. M., \& Ercole FF. (2017). Incidência e fatores de risco para infecção de sítio cirúrgico em cirurgias gerais. Rev. Latino-Am. Enfermagem, 25.

Costa, E. A. M. Moreira, L. L., \& Gusmão. (2019). Menincidence of infection of surgical site in hospital day: cohort of 74,213 patients monitored. Rev Sobecc,24(4), 211-216.

Costa, G. S. (2019). Propostas de melhoria nas ações de cuidado ao paciente, a partir do diagnóstico de infecções relacionadas à assistência à saúde (IRAS) em um hospital universitário de Fortaleza. - Dissertação (Mestrado) - Universidade Federal do Ceará, Programa de Pós-graduação em Políticas Públicas e Gestão da Educação Superior, Fortaleza (CE),

Cruz, L. A. Freitas, L. V. Barbosa, M. Gomes, L. F. S., \& Vasconcelos, C. M. T. (2013). Infección de herida operatoria tras cesárea en un hospital público de Fortaleza. Enfermeria Global, 29, 118-129.

Cunha, M. R. Padoveze, M. C. Melo, C. R. M., \& Nichiata, L. Y. I. (2018). Identification of post-cesarean surgical site infection: nursing consultation. Rev Bras enferm ., 71(3), 1478-86.

Feitosa, R. G. F. Fernandes, F. A. M. Junior, J. N. Junio, O. N. A. Costa, F. A., \& Cavalcante LDV. (2014). Analysis of the incidence of surgical site infection in oncologic surgeryof the digestive tract in Hospital Geral de Fortaleza. Rev. Medicina (Ribeirão Preto),47 (2), $157-64$.

Ferraz, C. C. B. Ortega, F. B. Silva, R. B. Leite, L. R. C., \& Hildebrand, C. R. (2016). Fatores associados a infecções hospitalares causadas por microrganismos multirresistentes num hospital de ensino. PECIBES, 2,52-57.

Lima, D. M. Wall, M. L. Hey, A. Falcade, A. C. Chaves, A. C. M., \& Souza, M. A. R. (2014). Fatores de riscos para infecção no puerpério cirúrgico. Cogitare Enferm.,19(4), 734-40. 
Research, Society and Development, v. 10, n. 14, e453101422241, 2021

(CC BY 4.0) | ISSN 2525-3409 | DOI: http://dx.doi.org/10.33448/rsd-v10i14.22241

Martins Filho, E. D. Santos, A. C. Rodrigues Junior, R. S. T. Adeodato, L. Coutinho, I., \& Katz L. (2010). Perfil epidemiológico e clínico de pacientes admitidas com diagnóstico de sepse puerperal de origem pélvica em uma UTI obstétrica no Nordeste do Brasil. Rev. Bras. Saude Mater. Infant.,10(4),469-475.

Martins, M. A. Goulart, E. M. A. França, E., \& Albert LR. (2012). Surgery infections in children and adolescents. Rev Med Minas Gerais, 22(3), 308-314.

MOTA, L. M. et al. (2010). Uso Racional de Antimicrobianos. Medicina, Ribeirão Preto, 43(2), 64-72.

Padoveze, M. C., \& Fortaleza, C. M. C. B. (2014). Healthcare-associated infections: challenges to public health in Brazil. Rev Saúde Pública, 48(6), 995-1001.

PARANÁ. (2018). Secretaria de Estado da Saúde (SESA). Linha guia rede mãe paranaense. Paraná.

Prates, C. G. Stadñik, C. M. B. Bagatini, A. Caregnato, R. C. A., \& Moura G. M. S. S. (2018). Comparación de las tasas de infección quirúrgica luego de implantación del checklist de seguridade. ACTA PAUL. ENFERM., 31(2), 116-122.

Santos, W. B. Araújo, M. G. S. Silva, J. C. Bernardo, T. H. L. Bastos, M. L. A., \& Verissimo, R. C. S. S. (2016). Surgical wounds infective microbiota: national and international analysis of scientific production. Rev Sobecc, 21(1), 46-51.

Say, L., et al. (2014). Global causes of maternal death: a WHO systematic analysis. Lancet Glob Health,2, 323-333.

Scardoni, A. Balzarini, F. Signorelli, C. Federico Cabitza, F., \& Odone, A. (2020). Artificial intelligence-based tools to control healthcare associatedinfections: A systematic review of the literature. Journal of Infection and Public Health, 13(8), 1061-1077

Souza, I. S. B. Santana, A. C., \& Júnior, G. D. (2018). The occurrence of surgical site infection: a review study. Rev. Med Minas Gerais,28(5), 168-175. 